\title{
A POLYTROPIC MODEL FOR SPACE AND LABORATORY PLASMAS DESCRIBED BY BI-MAXWELLIAN ELECTRON DISTRIBUTIONS
}

\author{
Yunchao Zhang, Christine Charles, and Rod Boswell \\ Space Plasma, Power and Propulsion Laboratory, Research School of Physics and Engineering, The Australian National University, \\ Bldg. 60, Mills Road, Canberra, ACT 2601, Australia; yunchao.zhang@ anu.edu.au \\ Received 2016 April 27; revised 2016 June 20; accepted 2016 July 13; published 2016 September 14
}

\begin{abstract}
Non-local electron energy probability functions (EEPFs) are shown to have an important effect on the thermodynamic behavior of plasmas in the context of solar wind and laboratory plasmas. A conservation relation is held for electron enthalpy and plasma potential during the electron transport. For an adiabatic system governed by non-local electron dynamics, the correlation between electron temperature and density can be approximated by a polytropic relation, with different indexes demonstrated using three cases of bi-Maxwellian EEPFs. This scenario differs from a local thermodynamic equilibrium having a single polytropic index of $5 / 3$ for adiabaticity.
\end{abstract}

Key words: methods: analytical - methods: data analysis - methods: laboratory: atomic - solar wind

\section{INTRODUCTION}

Astrophysical plasmas have been studied for many decades, including numerous work on the solar wind (Parker 1958; Meyer-Vernet 2007; Ofman 2010), a typical representation for stellar winds (Holzer \& Axford 1970; Cassinelli 1979; Kudritzki \& Puls 2000). Direct space measurements of the solar wind have been achieved using probes on board satellites (Scudder \& Olbert 1979; Sittler \& Scudder 1980; Doorsselaere et al. 2011). Additionally, many developments in understanding space phenomena can be linked and attributed to advances in theoretical modeling and experiments for laboratory plasmas (Koepke 2008). Hence an investigation of interrelation between the solar wind and laboratory plasmas can contribute to a better interpretation of the physics for both systems. Electrons in magnetically expanding low-pressure plasmas (Takahashi et al. 2009; Boswell et al. 2015) and in the solar wind share the following similarities: (1) they are confined along magnetic field lines; (2) they are nearly collisionless, due to the long mean free path in the lowpressure condition; (3) they have a thermal velocity greater than the plasma drift velocity, due to the small electron mass compared to that of ions; and (4) they are closely associated with a potential drop along the divergent magnetic field. The ions, however, demonstrate different behaviors in the solar wind and laboratory plasmas, but this does not affect the electron similarities stated above. For a laboratory plasma, the internal thermal energy of ions is small compared to their convective energy and they can be treated as a cold species for many cases (Tonks \& Langmuir 1929; Chabert \& Braithwaite 2011), while for the solar wind, the thermal energy of ions (mostly protons) is comparable to the convective energy and its effect is not negligible when determining the proton dynamics (Sittler \& Scudder 1980; Bruno \& Carbone 2013).

Since the mean velocity of a plasma flow is normally small compared to the electron velocity, the comoving frame attached to the flow can be considered as a stationary frame for electrons on first-order approximation, and this setting is used by default unless otherwise specified. When the electron energy and momentum relaxation paths are larger than the scale of the potential drop, the electrons move across the potential structure $(\phi(z)$, where $z$ is the spatial position) without encountering short-range electron-neutral collisions (including both elastic and inelastic collisions), and the kinetic energy term $(\epsilon)$ in the electron energy probability function (EEPF) $F_{p}(\epsilon, z)$ is replaced by the mechanical energy $\epsilon-e \phi(z)$, i.e., $F_{p}(\epsilon, z)=f_{p}[\epsilon-e \phi(z)]$. This generalized relation has been adopted for both space plasmas (Livadiotis 2015) and lowpressure laboratory plasmas (Tsendin 2009), and it is also known as the "non-locality" of EEPFs (Lieberman \& Lichtenberg 2005). When the pressure of neutral gases increases and the effect of electron-neutral collisions becomes dominant during electron transport, the "local" collision rate should be included in the expression of EEPF. A particle group dominated by collisions is considered to be under local thermodynamic equilibrium (LTE), which is the basis of traditional thermodynamic concepts such as the polytropic relation (Horedt 2004; Cengel \& Boles 2011). However, a collisionless or low-collisionality particle system, defined here as "non-LTE," behaves in a manner that is fundamentally different from that of an LTE system (Marsch 2006; Tsendin 2009): the mechanical energy of a non-LTE particle is conserved along its transport path, while that of an LTE particle is dissipated through collisions. Consequently, traditional thermodynamic properties based on LTE should be revisited for non-LTE plasmas (Livadiotis 2016).

A recent study (Zhang et al. 2016) has shown the role of non-local EEPFs in the thermodynamic behavior of plasma expansion in a laboratory helicon double layer thruster: the electron evolution along a potential drop in a divergent magnetic field is an adiabatic process and can be approximated by a polytropic relation with an index of 1.17 . This could be erroneously misinterpreted as the existence of thermal conduction from the surroundings into the system, since for traditional thermodynamic theory (Cengel \& Boles 2011) based on an LTE, an adiabatic process should have a polytropic index of $5 / 3$. Most interestingly, this polytropic index of 1.17 is consistent with that identified for the solar wind (Totten et al. 1995; Roussev et al. 2003; Jacobs \& Poedts 2011). Since the electrons in the solar wind and laboratory plasmas share important similarities, a reasonable hypothesis is that their polytropic relations are likely governed by the same principle of non-local EEPFs. Characterization of EEPFs along 

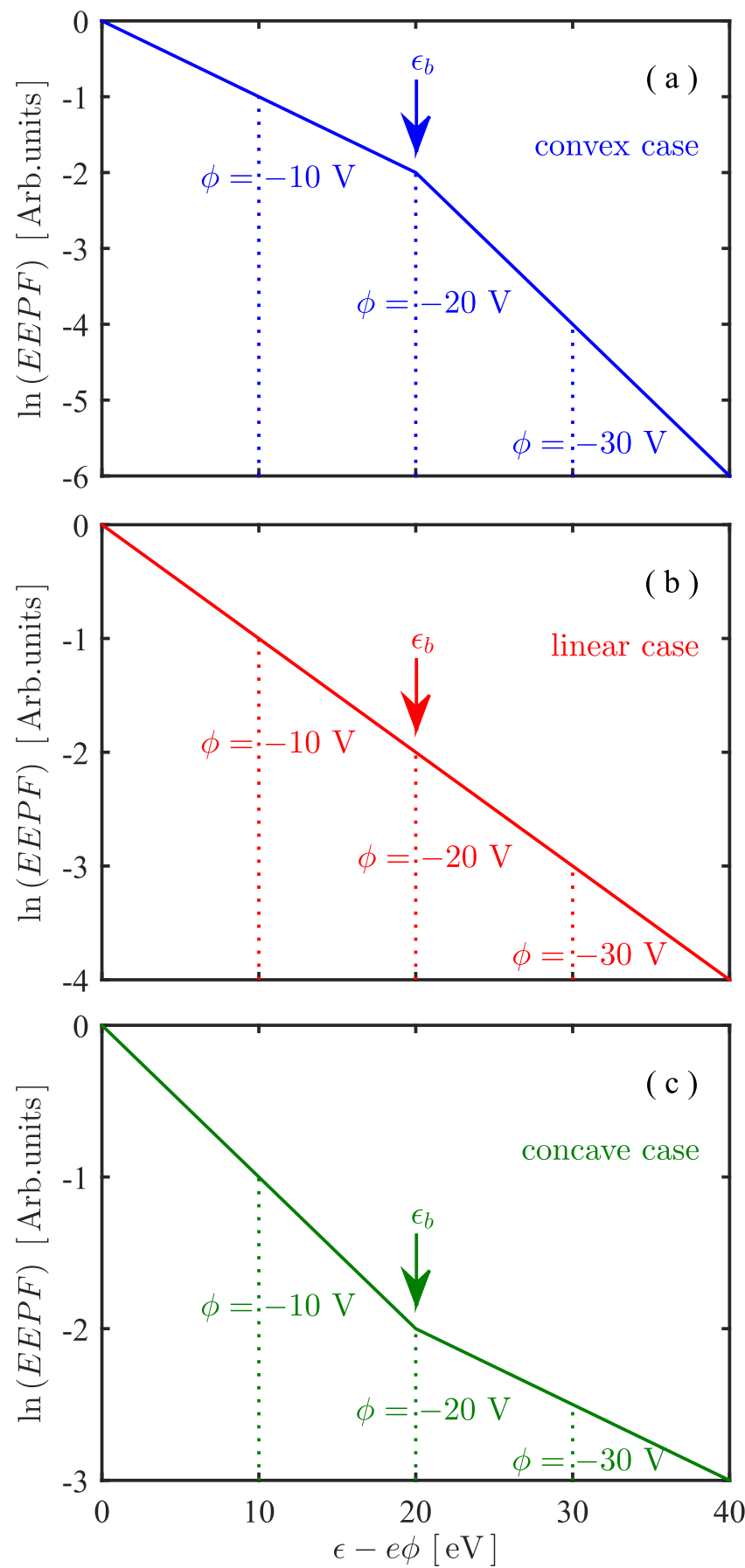

Figure 1. Three cases of bi-Maxwellian EEPFs: (a) the convex case $\left(T_{e 1}=10 \mathrm{eV}, T_{e 2}=5 \mathrm{eV}, c=\exp (4)\right), \quad$ (b) the linear case $\left(T_{e 1}=T_{e 2}=10 \mathrm{eV}, c=\exp (2)\right)$, and (c) the concave case $\left(T_{e 1}=10 \mathrm{eV}, T_{e 2}=20 \mathrm{eV}, c=\exp (1)\right)$, with a break energy of $\epsilon_{b}=20 \mathrm{eV}$. The origins of the EEPF curves at different potential locations are indicated by the vertical dashed lines.

the acceleration direction of the solar wind is currently unavailable and additional space measurements are still needed to verify this hypothesis. This study focuses on a new theoretical perspective of how non-local EEPFs determine the polytropic index of electrons. It should be noted that the polytropic approach has also been applied to protons, which is not discussed here but can be found in relevant literature (Kartalev et al. 2006; Nicolaou et al. 2014).

\section{ENTHALPY RELATION FOR NON-LOCAL ELECTRONS}

When electrons move non-locally along decreasing potentials, such as during plasma expanding along a divergent magnetic field (Boswell et al. 2015; Zhang et al. 2016), their transport is a self-consistent adiabatic process (the total mechanical energy of each electron is conserved) in which the EEPF is continuously depleted from low-energy electrons while high-energy electrons overcome the potential barrier. Electron enthalpy $h_{e}$ is defined using the formula $d h_{e}=d q_{e}+d p_{e} / n_{e}=d p_{e} / n_{e}$, with the heat term $d q_{e}$ omitted due to adiabaticity, where $p_{e}$ and $n_{e}$ are the electron pressure and density given by $p_{e}=2 / 3 \cdot \int_{0}^{\infty} \epsilon \sqrt{\epsilon} f_{p}(\epsilon-e \phi) d \epsilon$ and $n_{e}=\int_{0}^{\infty} \sqrt{\epsilon} f_{p}(\epsilon-e \phi) d \epsilon$, respectively. Integrating the above differential-form enthalpy along the potential path yields the enthalpy relation:

$$
\begin{aligned}
h_{e}= & \int_{-e \phi_{0}}^{-e \phi} \frac{\frac{2}{3} \int_{0}^{\infty} \epsilon \sqrt{\epsilon} f_{p}^{\prime}\left(\epsilon-e \phi_{x}\right) d \epsilon}{\int_{0}^{\infty} \sqrt{\epsilon} f_{p}\left(\epsilon-e \phi_{x}\right) d \epsilon} d\left(-e \phi_{x}\right) \\
& +h_{e 0}=e \phi-e \phi_{0}+h_{e 0},
\end{aligned}
$$

for which $\lim _{\epsilon \rightarrow \infty} \epsilon \sqrt{\epsilon} f_{p}\left(\epsilon-e \phi_{x}\right)=0$ has been used when applying integration by parts to the numerator.

Rearranging the above equation yields a conservation relation $\Delta h_{e}+\Delta(-e \phi)=0$, which shows that the electrons transfer their enthalpy into the potential energy in an adiabatic process. It should be noted that this conservation relation is a typical form of the Bernoulli integral (Kartalev et al. 2006; Livadiotis 2016), where the macro (convective) kinetic energy of the plasma flow is omitted due to the approximation of a stationary comoving frame as stated in the previous section. Equation (1) is a generalized result that is independent of the specific form of non-local EEPFs, and its differentiation with respect to the plasma transport path $z$ yields:

$$
\frac{d p_{e}}{d z}+n_{e} E_{z}=0
$$

where $E_{z}$ is the electric field along the $z$ direction. Equation (2) has a consistent form of momentum balance with a LTE system, but the pressure term here is an effective parameter determined by non-local motion of electrons rather than by local collisions. Previous experiments in laboratory plasmas (Zhang et al. 2016) have verified enthalpy relation (1) for electrons along a divergent magnetic field. It should be noted that for solar electrons covering large distances, the electron enthalpy will be partially consumed in order to overcome the gravitational barrier of the Sun.

\section{POLYTROPIC APPROXIMATION}

Although the enthalpy relation is independent of the specific form of non-local EEPFs, another important thermodynamic property, the "polytropic relation" (widely used to characterize processes involving energy exchange between a system and its surrounding environment) is shown to depend on the shape of EEPFs in an adiabatic system using the example of a typical 
bi-Maxwellian EEPF:

$$
f_{p}(\epsilon-e \phi)= \begin{cases}c \cdot \exp \left(-\frac{\epsilon_{b}}{T_{e 2}}-\frac{\epsilon-e \phi}{T_{e 1}}\right), & \epsilon-e \phi<\epsilon_{b} \\ c \cdot \exp \left(-\frac{\epsilon_{b}}{T_{e 1}}-\frac{\epsilon-e \phi}{T_{e 2}}\right), & \epsilon-e \phi>\epsilon_{b},\end{cases}
$$

where $c, \epsilon_{b}, T_{e 1}$, and $T_{e 2}$ are the scale coefficient, break energy, first electron temperature for a low-energy range and second electron temperature for a high-energy range, respectively. For the present demonstration, we use a break energy of $\epsilon_{b}=20 \mathrm{eV}$, a first electron temperature of $T_{e 1}=10 \mathrm{eV}$, and three different values for the second electron temperature $T_{e 2}=5,10,20 \mathrm{eV}$, which result in "convex," "linear," and "concave" EEPF curves as a function of $\epsilon-e \phi$ (Figures 1(a), (b), and (c), respectively). For simplicity, the scale coefficient $c$ for each case is chosen to have $f_{p}(0)=1$ and the initial maximum potential is set to zero $\phi_{0}=0$, with the reminding range satisfying $\phi \leqslant 0$. The EEPFs at different potential locations are represented by the curves with right-shifting origins (vertical dashed lines) and their non-locality is reflected by the amplitude consistency. The plasma parameters of electron pressure, electron density, and electron temperature are calculated from the bi-Maxwellian EEPFs, given by

$$
\begin{aligned}
\frac{p_{e}}{c}= & {\left[\frac{2}{3}\left(e \phi+\epsilon_{b}\right)+T_{e 1}+T_{e 2}\right]\left(e \phi+\epsilon_{b}\right)^{\frac{1}{2}} } \\
& \times\left(T_{e 2}-T_{e 1}\right) \exp \left(-\frac{\epsilon_{b}}{T_{e 1}}-\frac{\epsilon_{b}}{T_{e 2}}\right) \\
& +\frac{\pi^{\frac{1}{2}} T_{e 1}^{\frac{5}{2}}}{2} \exp \left(\frac{e \phi}{T_{e 1}}-\frac{\epsilon_{b}}{T_{e 2}}\right) \operatorname{erf}\left[\left(\frac{e \phi+\epsilon_{b}}{T_{e 1}}\right)^{\frac{1}{2}}\right] \\
& +\frac{\pi^{\frac{1}{2}} T_{e 2}^{\frac{5}{2}}}{2} \exp \left(\frac{e \phi}{T_{e 2}}-\frac{\epsilon_{b}}{T_{e 1}}\right) \operatorname{erfc}\left[\left(\frac{e \phi+\epsilon_{b}}{T_{e 2}}\right)^{\frac{1}{2}}\right], \\
\frac{n_{e}}{c}= & \left(e \phi+\epsilon_{b}\right)^{\frac{1}{2}}\left(T_{e 2}-T_{e 1}\right) \exp \left(-\frac{\epsilon_{b}}{T_{e 1}}-\frac{\epsilon_{b}}{T_{e 2}}\right) \\
& +\frac{\pi^{\frac{1}{2}} T_{e 1}^{\frac{3}{2}}}{2} \exp \left(\frac{e \phi}{T_{e 1}}-\frac{\epsilon_{b}}{T_{e 2}}\right) \operatorname{erf}\left[\left(\frac{e \phi+\epsilon_{b}}{T_{e 1}}\right)^{\frac{1}{2}}\right] \\
& +\frac{\pi^{\frac{1}{2}} T_{e 2}^{\frac{3}{2}}}{2} \exp \left(\frac{e \phi}{T_{e 2}}-\frac{\epsilon_{b}}{T_{e 1}}\right) \operatorname{erfc}\left[\left(\frac{e \phi+\epsilon_{b}}{T_{e 2}}\right)^{\frac{1}{2}}\right] \\
& T_{e}=\frac{p_{e}}{n_{e}} .
\end{aligned}
$$

These expressions are valid for potential values satisfying $\phi \geqslant-\epsilon_{b} / e$. For $\phi<-\epsilon_{b} / e$ the electrons are located in the Maxwellian region (as illustrated by the EEPF at $\phi=-30 \mathrm{~V}$ in Figure 1) and the corresponding results are simply given by the Boltzmann-type relations:

$$
\frac{p_{e}}{c}=\frac{\pi^{\frac{1}{2}} T_{e 2}^{\frac{5}{2}}}{2} \exp \left(\frac{e \phi}{T_{e 2}}-\frac{\epsilon_{b}}{T_{e 1}}\right),
$$


Figure 2. (a) Electron pressure, (b) electron density, and (c) electron temperature as a function of potential for three EEPF cases: the convex case (open circles, $T_{e 1}=10 \mathrm{eV}, T_{e 2}=5 \mathrm{eV}, c=\exp (4)$ ), the linear case (open squares, $T_{e 1}=T_{e 2}=10 \mathrm{eV}, c=\exp (2)$ ), and the concave case (open triangles, $\left.T_{e 1}=10 \mathrm{eV}, T_{e 2}=20 \mathrm{eV}, c=\exp (1)\right)$, with a break energy of $\epsilon_{b}=20 \mathrm{eV}$.

$$
\begin{gathered}
\frac{n_{e}}{c}=\frac{\pi^{\frac{1}{2}} T_{e 2}^{\frac{3}{2}}}{2} \exp \left(\frac{e \phi}{T_{e 2}}-\frac{\epsilon_{b}}{T_{e 1}}\right), \\
T_{e}=T_{e 2} .
\end{gathered}
$$

It should be noted that for other forms of EEPFs, analytical expressions for the above parameters may not be available and numerical approximation should be used.

The results of electron pressure, electron density, and electron temperature versus potential are respectively given in Figures 2(a)-(c) for the "convex," "linear," and "concave" 

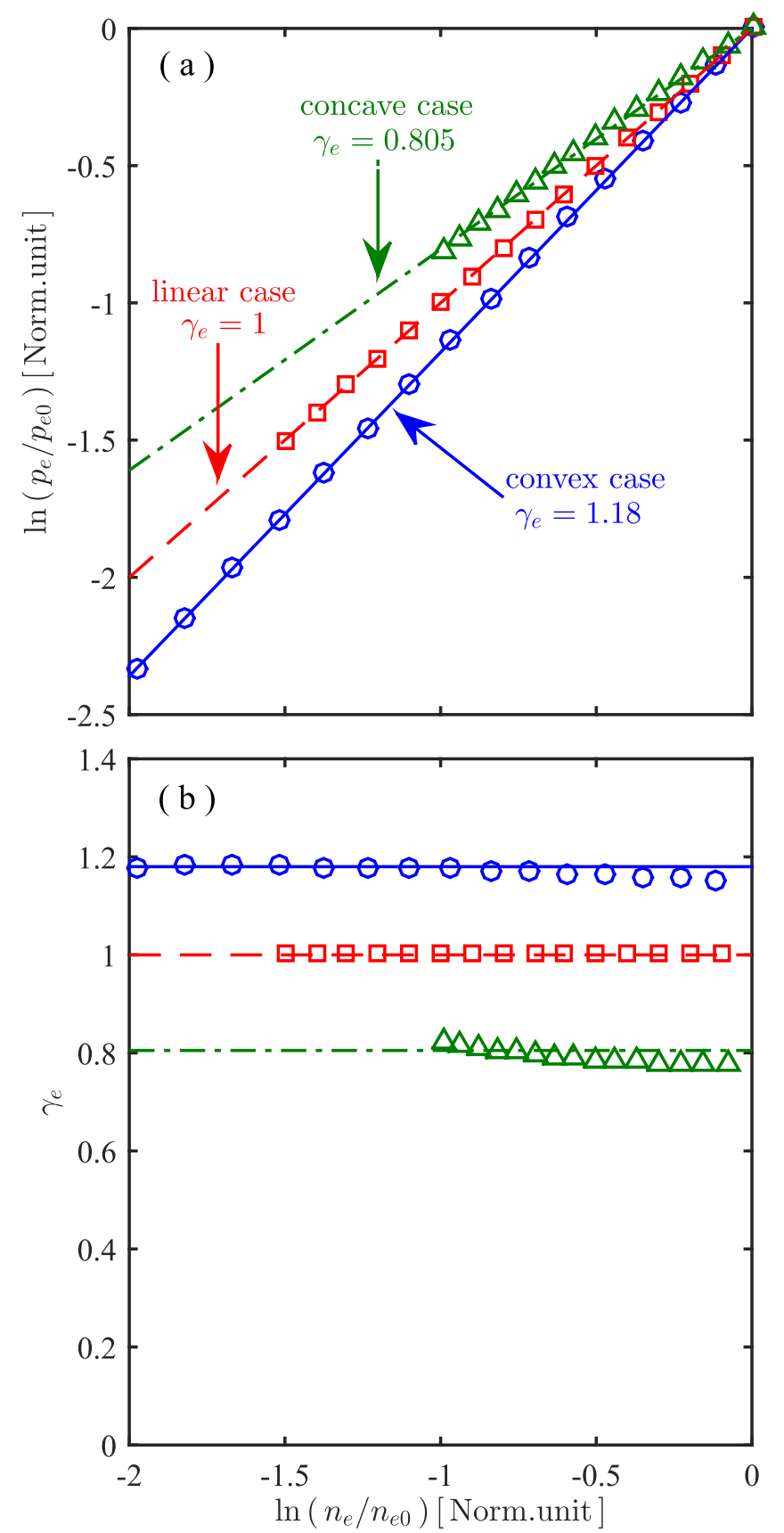

Figure 3. (a) Correlation data between the logarithm of normalized electron density and the logarithm of normalized electron pressure in a potential range of $-15 \mathrm{~V}<\phi<0 \mathrm{~V}$, and (b) polytropic indices given by the ratio of $\log \left(p_{e} / p_{e 0}\right)$ to $\log \left(n_{e} / n_{e 0}\right)$ at different data points, for three EEPF cases: the convex (open circles, $T_{e 1}=10 \mathrm{eV}, T_{e 2}=5 \mathrm{eV}, c=\exp (4)$ ), linear (open squares, $T_{e 1}=T_{e 2}=10 \mathrm{eV}, c=\exp (2)$ ), and concave (open triangles, $\left.T_{e 1}=10 \mathrm{eV}, T_{e 2}=20 \mathrm{eV}, c=\exp (1)\right)$ cases, with a break energy of $\epsilon_{b}=20 \mathrm{eV}$. Their respective polytropic relations are fitted with indexes of 1.18 (solid line), 1 (dashed line), and 0.805 (dash-dotted line).

EEPFs. The electron pressure and density decrease most dramatically for the convex EEPFs (open circles) and decrease to a lesser degree for the concave EEPFs (open triangles). For $\phi<-\epsilon_{b} / e$ the three cases show a constant temperature, while for $\phi>-\epsilon_{b} / e$ the electron temperature decreases for the convex EEPFs and increases for the concave EEPFs.

A correlation between the logarithm of normalized electron density $\log \left(n_{e} / n_{e 0}\right)$ and the logarithm of normalized electron pressure $\log \left(p_{e} / p_{e 0}\right)$, where the subscript " 0 " indicates parametric values at the position of the zero plasma potential, is shown in Figure 3(a) for the three EEPF cases in the potential range of $-15 \mathrm{~V}<\phi<0 \mathrm{~V}$ (data from Figures 2(a) and (b)). This data can be fitted by the polytropic relation $\log \left(p_{e} / p_{e 0}\right)=\gamma_{e} \cdot \log \left(n_{e} / n_{e 0}\right)$, where $\gamma_{e}$ is the polytropic index for electrons, with results for the convex EEPFs fitted by a polytropic curve with an index of $\gamma_{e}=1.18$ (solid line), the linear EEPFs with $\gamma_{e}=1$ (dashed line), and the concave EEPFs with $\gamma_{e}=0.805$ (dashed-dotted line). Figure 3(b) shows the polytropic index $\gamma_{e}$ as a function of $\log \left(n_{e} / n_{e 0}\right)$ given by the ratio of $\log \left(p_{e} / p_{e 0}\right)$ to $\log \left(n_{e} / n_{e 0}\right)$ at different data points on Figure 3(a), and for each EEPF case $\gamma_{e}\left[\log \left(n_{e} / n_{e 0}\right)\right]$ presents a relatively constant value that is consistent with their respective fitted indices represented by horizontal lines. It should be noted that the polytropic relation is an approximation method used to describe a thermodynamic process rather than an exact model, and hence a perfect fitting between the sampled data and polytropic curve is not always expected. The polytropic index depends on the specific shape of EEPFs, increasing when the EEPF curves become more convex and decreasing when they are more concave. Additionally, a typical case of convex nonlocal EEPFs with a rectangular shape is constructed as follows: the non-local probability function is constructed using the Heaviside function (denoted by $H$ ) to give $f_{p}(\epsilon-e \phi)=c\left[H\left(2.5 T_{e 0}+e \phi-\epsilon\right)-H(e \phi-\epsilon)\right]$, where $c$ is the scale coefficient and the plasma potential satisfies $\phi \leqslant 0$. In this case the EEPFs present a sequence of depleted rectangles along the potential drop and result in an exact polytropic index of $\gamma_{e}=5 / 3$.

Hence, for an adiabatic process governed by non-local EEPFs, multiple polytropic index values can be achieved, as illustrated above using bi-Maxwellian EEPFs with $\gamma_{e}=1.18,1,0.805$ and rectangular EEPFs with $\gamma_{e}=5 / 3$. Using traditional thermodynamics based on LTE would misinterpret the adiabatic processes with $\gamma_{e}<5 / 3$ as additional heat being brought into the system. The classic adiabatic index of 5/3 for LTE systems is only an element in the set of polytropic indexes for non-LTE adiabatic systems. These results suggest that although the electrons in the solar wind present a polytropic index of less than $5 / 3$, as previously reported (Sittler \& Scudder 1980; Doorsselaere et al. 2011), their actual transport could be an adiabatic process.

\section{SUMMARY}

This paper revisits the thermodynamic behaviors of plasmas in the solar wind and in the laboratory. As electrons in the two systems share many similarities, they may be dominated by the same principle of non-local EEPFs. For an adiabatic evolution of electrons along a potential path, a conservation relation between the electron enthalpy and plasma potential has been found. Correlation between the electron temperature and density can be approximated by the polytropic relation for which three typical cases of bi-Maxwellian EEPFs with convex, linear, and concave shapes have been treated. Multiple polytropic indexes can be achieved during an adiabatic process depending on the specific shape of non-local EEPFs; the polytropic index increases when the EEPF becomes more convex and decreases when the EEPF becomes more concave. The classic adiabatic index of 5/3 for LTE systems is only one element in the set of polytropic indexes for non-LTE adiabatic systems governed by non-local particles. These results suggest 
that although the electrons in the solar wind have a polytropic index of less than $5 / 3$, their actual transport might be adiabatic.

\section{REFERENCES}

Boswell, R. W., Takahashi, K., Charles, C., \& Kaganovich, I. D. 2015, FrPhy, 3, 14 Bruno, R., \& Carbone, V. 2013, LRSP, 10, 2

Cassinelli, J. P. 1979, ARA\&A, 17, 275

Çengel, Y. A., \& Boles, M. A. 2011, Thermodynamics: An Engineering Approach (7th ed.; New York: McGraw-Hill)

Chabert, P., \& Braithwaite, N. 2011, Physics of Radio-Frequency Plasmas (Cambridge: Cambridge Univ. Press)

Doorsselaere, T. V., Wardle, N., Zanna, G. D., et al. 2011, ApJL, 727, L32

Holzer, T. E., \& Axford, W. I. 1970, ARA\&A, 8, 31

Horedt, G. P. 2004, Polytropes: Applications in Astrophysics and Related Fields (New York: Kluwer)

Jacobs, C., \& Poedts, S. 2011, AdSpR, 48, 1958

Kartalev, M., Dryer, M., Grigorov, K., \& Stoimenova, E. 2006, JGR, 111, A10107

Koepke, M. E. 2008, RvGeo, 46, RG3001
Kudritzki, R. P., \& Puls, J. 2000, ARA\&A, 38, 613

Lieberman, M. A., \& Lichtenberg, A. J. 2005, Principles of Plasma Discharges and Materials Processing (2nd ed.; New York: Wiley)

Livadiotis, G. 2015, JGRA, 120, 880

Livadiotis, G. 2016, ApJS, 223, 13

Marsch, E. 2006, LRSP, 3, 1

Meyer-Vernet, N. 2007, Basics of the Solar Wind (New York: Cambridge Univ. Press)

Nicolaou, G., Livadiotis, G., \& Moussas, X. 2014, SoPh, 289, 1371

Ofman, L. 2010, LRSP, 7, 4

Parker, E. N. 1958, ApJ, 128, 664

Roussev, I. I., Gombosi, T. I., Sokolov, I. V., et al. 2003, ApJL, 595, L57

Scudder, J. D., \& Olbert, S. 1979, JGR, 84, 6603

Sittler, E. C., \& Scudder, J. D. 1980, JGR, 85, 5131

Takahashi, K., Charles, C., Boswell, R. W., Cox, W., \& Hatakeyama, R. 2009, ApPhL, 94, 191503

Tonks, L., \& Langmuir, I. 1929, PhRv, 34, 876

Totten, T. L., Freeman, J. W., \& Arya, S. 1995, JGR, 100, 13

Tsendin, L. D. 2009, PSST, 18, 014020

Zhang, Y., Charles, C., \& Boswell, R. W. 2016, PhRvL, 116, 025001 